\title{
Geometric Parameter Identification of a 6-DOF Space Robot Using a Laser-Ranger
}

\author{
Yu Liu, ${ }^{1}$ Zainan Jiang, ${ }^{1}$ Hong Liu, ${ }^{1}$ and Wenfu $\mathrm{Xu}^{2}$ \\ ${ }^{1}$ State Key Laboratory of Robotics and System, Harbin Institute of Technology, Harbin 150001, China \\ ${ }^{2}$ Mechanical Engineering and Automation, Harbin Institute of Technology Shenzhen Graduate School, Shenzhen 518057, China
}

Correspondence should be addressed to Yu Liu, lyu11@hit.edu.cn

Received 27 December 2011; Revised 7 March 2012; Accepted 8 March 2012

Academic Editor: Zhuming Bi

Copyright ( $2012 \mathrm{Yu}$ Liu et al. This is an open access article distributed under the Creative Commons Attribution License, which permits unrestricted use, distribution, and reproduction in any medium, provided the original work is properly cited.

\begin{abstract}
The geometric parameters of a space robot change with the terrible temperature change in orbit, which will cause the end-effector pose (position and orientation) error of a space robot, and so weakens its operability. With this in consideration, a new geometric parameter identification method is presented based on a laser-ranger attached to the end-effector. Then, independence of the geometric parameters is analyzed, and their identification equations are derived. With the derived identification Jacobian matrix, the optimal identification configurations are chosen according to the observability index $\mathrm{O}_{3}$. Subsequently, through simulation the geometric parameter identification of a 6-DOF space robot is implemented for these identification configurations, and the identified parameters are verified in a set of independent reference configurations. The result shows that in spite of distance measurement alone, pose accuracy of the space robot still has a greater improvement, so the identification method is practical and valid.
\end{abstract}

\section{Introduction}

Repeatability of the robot only represents the ability that the robot follows the same trajectory or gets to the same desired poses time after time, so it more indicates compactness of the robot. Comparatively, pose accuracy of the robot describes how close the end effector true pose is to desired pose. Good repeatability is the premise of high accuracy for a robot. Generally, for such simple tasks as conveying goods, spraying paint, or welding an automobile, high repeatability is already enough, because these jobs can be completed through teaching and playback. However, in some other occasions, for example, the medical robot bores a hole on the bone for a patient with the aid of X-ray image, or more typically the space robot guided by hand-eye vision maintains a faulty space vehicle, in this case it is necessary to map the end effector Cartesian coordinates into the joint coordinates, namely, the joint angles must be evaluated through inverse kinematics. However, subject to difference between the nominal geometrical parameters of the robot links and their true parameters, the calculated joint angles do not correspond with the desired ones, which cause the end effector pose errors. At the same time, pose errors may result from nongeometrical errors, for example, joint and link deformation, transmission, and temperature.

Consequently, the robot kinematic parameter identification must be done to improve the end effector pose accuracy before it is used. Virtually, parameter identification is a software compensation algorithm, because it only seeks for the true kinematic parameters and does not physically change the links, joints, and controllers of the robot. It can be divided into two categories, that is, geometrical parameter identification and nongeometrical one. Most researchers concentrate on the former. Veitschegger and wu [1] developed a method of kinematic calibration and compensation, and with the least square algorithm calibrated the PUMA 560 experimentally. The experiment results showed that a greater than 70 times improvement in Cartesian pose errors resulted from the calibrated versus the nominal manipulator. Stone et al. $[2,3]$ modeled kinematics errors using a six-parameter "Smodel" per link, then they introduced three features of the robot to estimate the 6n S-model parameters. Lukas Beyer and Wulfsberg [4] developed an ROSY calibration system with two CCD cameras and a reference sphere that enabled 
pose accuracy to be improved for conventional arms and parallel robots. Sun and Hollerbach [5] presented an active robot calibration algorithm using the determinant-based updating observability index and demonstrated it through simulation with a 6-DOF PUMA 560 robot. Kang et al. [6] introduced a new metrology method based on the productof-exponential formula and the modified dyad kinematics to calibrate a modular robot, but there were no calibration results to be given.

Research on nongeometrical parameter identification has also made great progress. Chen and Chao [7] presented a sixparameter error model between two consecutive links in a general sense and developed a mathematical identification model composed of nongeometrical parameters, it considered the second, the third joint, and the link flexibility due to gravity. Judd and Knasinski [8] analyzed nongeometrical errors (gear train errors, joint, and link flexibility, etc.) and proposed an error model that can be identified with a common least squares procedure. Chunhe Gong et al. [9] built a comprehensive error model including geometric errors, position-dependent compliance errors, and time-variant thermal errors, and robot accuracy was improved by an order of magnitude after calibration. Lightcap et al. [10] applied a 30-parameter flexible geometric model to the Mitsubishi PA10-6CE robot, considering the flexibility in the harmonic drive transmission. Drouet et al. [11] decomposed the measured end-point error into generalized geometric and elastic errors and realized compensation for dynamic elastic effects. With a camera attached to the end effector, Radkhah et al. [12] used an extended forward kinematic model incorporating both geometric and nongeometric parameters to identify the KUKA KR 125/2 robot kinematic parameters.

Space robots lie in microgravity environments and move slowly, so nongeometrical errors due to joint and link flexibility will occupy a small proportion, and here they are omitted. However, subject to extreme temperature under space environment, the geometric parameters of space robots will have a great change. The extravehicular temperature scope in orbit is $\pm 80^{\circ} \mathrm{C}$ or so, and the inner temperature scope of the space robot is $-30^{\circ} \mathrm{C} \sim+50^{\circ} \mathrm{C}$ under the condition of temperature control. For a two-meter robotic arm, its maximum length variation is $2 \mathrm{~mm}$ or so. Besides, there is a temperature difference between the lighted surface of the space robot and the shady surface, which will cause deformation of the space robot. So, a space robot calibrated on the ground must be recalibrated on orbit to improve its pose accuracy. Sometimes, the space robot will carry a laserranger attached to its end-effector to detect the manipulated objects [13, 14], using it the paper will discuss geometric parameter identification of the on-orbit space robot, and give the simulation results.

\section{Kinematic Model of the Space Robot}

2.1. Outline of Identification Scheme. As shown in Figure 1, the space robot is fixed on the $+Z$ surface (pointing to the center of the earth) of the satellite, and its end-effector carries a laser-ranger that is used to measure the distance from the

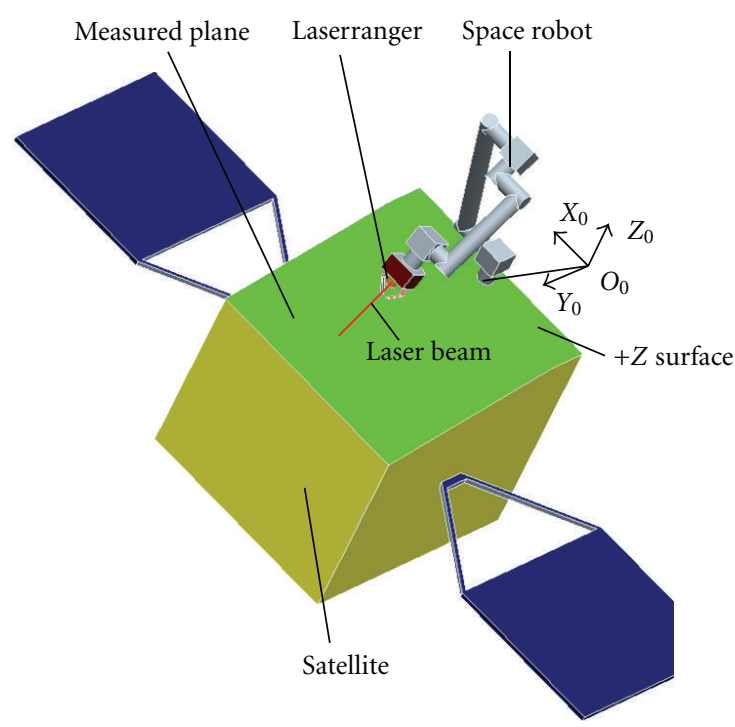

FIGURE 1: Sketch of parameter identification scheme of the space robot.

starting point of the laser beam to the measured declining plane. Because the equation of the plane with respect to the base coordinate frame is known and the starting point and the equation of the laser beam (line) with respect to the tool frame can be calibrated beforehand, so the distance can also be estimated according to the kinematic model. However, the model is inaccurate because of the geometric parameter errors of the space robot, so there exists the difference between the measured distance and that calculated with the nominal kinematic parameters, which is used to identify the geometric parameters of the space robot. Some other parameter identification methods [15-17] using a laser-ranger generally measured the distance from the robot end-point to a known object point, however, it was difficult to determine whether the laser beam just passed through the object point in practice. In the literature [15], the position-sensitive detector (PSD) was adopted, which increased complexity of parameter identification. Here, the known declining plane is chosen as the object measured by the laser-ranger, which simplifies the measurement scheme. In the literature [17], the laser spot was measured by a camera, which introduced measurement noise of the camera.

2.2. Kinematic Model. Commonly, with the D-H parameter method, the relative translation and rotation from the robot link frame $i-1$ to the frame $i$ can be described by a homogeneous transformation matrix ${ }^{i-1} \mathbf{A}_{i}$ as

$$
{ }^{i-1} \mathbf{A}_{i}=\left[\begin{array}{cccc}
C \theta_{i} & -C \alpha_{i} S \theta_{i} & S \alpha_{i} S \theta_{i} & a_{i} C \theta_{i} \\
S \theta_{i} & C \alpha_{i} C \theta_{i} & -S \alpha_{i} \cos \theta_{i} & a_{i} S \theta_{i} \\
0 & S \alpha_{i} & C \alpha_{i} & d_{i} \\
0 & 0 & 0 & 1
\end{array}\right],
$$

where, $C \theta_{i}$ denotes $\cos \left(\theta_{i}\right), S \theta_{i}$ represents $\sin \left(\theta_{i}\right)$, and the rest may be deduced by analogy. ${ }^{i-1} \mathbf{A}_{i}$ includes four kinematic parameters, namely $\theta_{i}, d_{i}, a_{i}$, and $\alpha_{i}$. However, when a small angle variation creates between two consecutive parallel axes 
or near parallel axes, with the D-H method, it will lead to a large variation of the parameter $d_{i}$, in other words, in this case the axial offset $d_{i}$ is very sensitive to the twist $\alpha_{i}$. In view of this, the matrix ${ }^{i-1} \mathbf{A}_{i}$ is post-multiplied the matrix ${ }^{i-1} \mathbf{A}_{i}$ by an additional rotational matrix $\operatorname{Rot}\left(y, \beta_{i}\right)$ [18], namely the matrix ${ }^{i-1} \mathbf{A}_{i}$ can be changed as

$$
{ }^{i-1} \mathbf{A}_{i} \leftarrow{ }^{i-1} \mathbf{A}_{i} \cdot \operatorname{Rot}\left(y, \beta_{i}\right)=\left(\begin{array}{cccc}
C \theta_{i} C \beta_{i}-S \theta_{i} S \alpha_{i} S \beta_{i} & -S \theta_{i} C \alpha_{i} & C \theta_{i} S \beta_{i}+S \theta_{i} S \alpha_{i} S \beta_{i} & a_{i} C \theta_{i} \\
S \theta_{i} C \beta_{i}+C \theta_{i} S \alpha_{i} S \beta_{i} & C \theta_{i} C \alpha_{i} & S \theta_{i} S \beta_{i}-C \theta_{i} S \alpha_{i} C \beta_{i} & a_{i} S \theta_{i} \\
-C \alpha_{i} S \beta_{i} & S \alpha_{i} & C \alpha_{i} C \beta_{i} & d_{i} \\
0 & 0 & 0 & 1
\end{array}\right),
$$

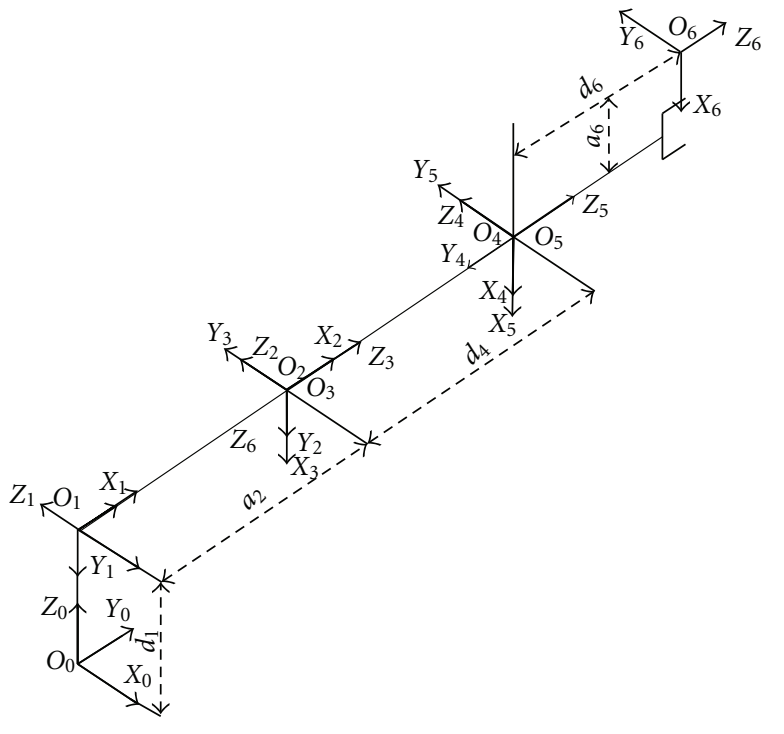

FIGURE 2: Representation of the coordinate frames of the space robot with $\mathrm{D}-\mathrm{H}$ convention.

where

$$
\operatorname{Rot}\left(y, \beta_{i}\right)=\left[\begin{array}{cccc}
C \beta_{i} & 0 & S \beta_{i} & 0 \\
0 & 1 & 0 & 0 \\
-S \beta_{i} & 0 & C \beta_{i} & 0 \\
0 & 0 & 0 & 1
\end{array}\right]
$$

Supposed that ${ }^{0} \mathbf{A}_{1}$ represents the transformation matrix from the base coordinate frame to the frame 1 , in terms of Figure 2, the transformation matrix $\mathbf{T}_{N}$ from the base coordinate frame to the tool frame can be obtained from the well known loop closure equation:

$$
\mathbf{T}_{N}={ }^{0} \mathbf{A}_{n}={ }^{0} \mathbf{A}_{1}{ }^{1} \mathbf{A}_{2} \ldots{ }^{5} \mathbf{A}_{6} .
$$

Further, the matrix $\mathbf{T}_{N}$ can be divided into the following submatrix:

$$
\mathbf{T}_{N}=\left(\begin{array}{cc}
\mathbf{R}_{N} & \mathbf{p}_{N} \\
0 & 1
\end{array}\right)
$$

where $\mathbf{R}_{N} \in R^{3 \times 3}$ is an orientation matrix of the tool frame with respect to the base frame, $\mathbf{p}_{N} \in R^{3}$ denotes the translational vector.

The configuration of the space robot is shown in Figure 2, the tool frame $O_{6}-X_{6} Y_{6} Z_{6}$ of the space robot can be chosen arbitrarily. Here, we might as well choose the laser-ranger coordinate frame fixed to the end-effector as the tool frame, namely, the starting point of the laser beam is located in the origin $O_{6}$ and the positive direction of the $Z_{6}$ axis acts as the emission direction of the laser beam, which helps to simplify identification process and decrease the complexity of robot identification.

\section{Identification Model of the Geometric Parameters}

3.1. Independent Parameters of the Identification Model. A complete kinematic model consists of a certain number of the independent parameters. If the model exceeds the scope, they will be relative. Therefore, extra increment of the number of the parameters is insignificant to improvement of pose accuracy. Everett et al. [19] gave the following calculative formula:

$$
C=4 R+2 P+6,
$$

where, $C$ denotes the number of independent parameters (also constrained equations), $R$ is the number of the revolved joints, and $P$ is the number of the translational joints. Besides, Figure 6 of (6) represents 6 constraints that determine the pose of the tool frame with respect to the link 5 frame $\mathrm{O}_{5}-\mathrm{X}_{5} Y_{5} Z_{5}$. According to the above equation, the space robot shown in Figure 2 totally has 30 independent geometric parameters. However, different from a laser tracker to measure a 6-dimension pose of the robot, the laserranger only measures the distance from the origin of the laserranger coordinate frame to the objective point. Obviously, an arbitrary equal-distance rotation of the end-effector around the target point creates no significance to output of the laserranger, in other words, in the spherical surface whose spherical center is the target point, and its radius is the measured distance, however, the tool coordinate frame moves, the measured distance is same, and it means that the orientation of the end-effector cannot be constrained. In addition, a distance equation only constrains one of the three coordinates for a point, while the other two coordinates are free. Namely, compared with the laser tracker, the laserranger loses five constraints, and maximally there are 25 identifiable parameters for the space robot.

3.2. Identification Equation. According to (5) and the above laser-ranger coordinate frame, it is easy to know that 
the starting point $\mathbf{p}_{s}$ of the laser beam with respect to the base coordinate frame is equivalent to the translational vector $\mathbf{p}_{n}$.

Similarly, the laser beam unit vector $\mathbf{b}_{l}$ relative to the base coordinate frame is expressed as

$$
\mathbf{b}_{l}=\mathbf{R}_{N}\left[\begin{array}{l}
0 \\
0 \\
1
\end{array}\right] .
$$

It is assumed that the measured plane equation in the base coordinate frame is

$$
\mathbf{n}_{l} \cdot \mathbf{p}+f=0
$$

where $\mathbf{n}_{l}\left(n_{l x}, n_{l y}, n_{l z}\right)$ is the unit normal vector of the measured plane, its positive direction can be chosen arbitrarily, here, $n_{l z}$ is given a positive value. $\mathbf{p}$ denotes the coordinate vector $\left(p_{x}, p_{y}, p_{z}\right)$ of the arbitrary point in the plane, and $f$ is a known scalar. Supposed that the laser beam vector $\mathbf{b}_{l}$ intersects the measured plane at the point $\mathbf{p}_{j}$, then according to the relation of the vectors, $\mathbf{p}_{j}$ can be written as

$$
\mathbf{p}_{j}=\mathbf{p}_{s}+h \mathbf{b}_{l},
$$

where $h$ denotes the distance from the starting point $\mathbf{p}_{s}$ of the laser beam to the intersectant point $\mathbf{p}_{j}$. As We know, $\mathbf{p}_{j}$. Also meets (8), then by substituting (9) into (8) $h$ can be expressed as

$$
h=-\frac{\mathbf{n}_{l} \cdot \mathbf{p}_{s}+f}{\mathbf{n}_{l} \cdot \mathbf{b}_{l}} .
$$

The distance $h$ in (10) is an estimated value based on the nominal geometric parameters of the space robot and the nominal plane equation. As stated previously, these geometric parameters on space orbit generally deviate from the nominal ones. The geometric errors in the link $i$ are. respectively, written as $\Delta \theta_{i}, \Delta d_{i}, \Delta a_{i}, \Delta \alpha_{i}$, and $\Delta \beta_{i}$. Here, it is assumed that they are small amount, so a linear model can be developed for simplicity. If the true parameters of the link $i$ are, respectively, given as $\theta_{i}^{r}, d_{i}^{r}, a_{i}^{r}, \alpha_{i}^{r}$, and $\beta_{i}^{r}$, there are the following relations:

$$
\begin{gathered}
\theta_{i}^{r}=\theta_{i}+\Delta \theta_{i} \quad d_{i}^{r}=d_{i}+\Delta d_{i}, \\
a_{i}^{r}=a_{i}+\Delta a_{i} \cdots \alpha_{i}^{r}=\alpha_{i}+\Delta \alpha_{i}, \quad \beta_{i}^{r}=\beta_{i}+\Delta \beta_{i} .
\end{gathered}
$$

Differentiate (10), then

$$
\begin{aligned}
\Delta h= & h^{r}-h \\
\approx & \frac{\partial h}{\partial \theta_{1}} \Delta \theta_{1}+\frac{\partial h}{\partial d_{1}} \Delta d_{1}+\frac{\partial h}{\partial a_{1}} \Delta a_{1}+\frac{\partial h}{\partial \alpha_{1}} \Delta \alpha_{1} \\
& +\frac{\partial h}{\partial \beta_{1}} \Delta \beta_{1}+\cdots+\frac{\partial h}{\partial \theta_{6}} \Delta \theta_{6}+\frac{\partial h}{\partial d_{6}} \Delta d_{6}+\frac{\partial h}{\partial a_{6}} \Delta a_{6} \\
& +\frac{\partial h}{\partial \alpha_{6}} \Delta \alpha_{6}+\frac{\partial h}{\partial \beta_{6}} \Delta \beta_{6}+\frac{\partial h}{\partial n_{l y}} \Delta n_{l y}+\frac{\partial h}{\partial n_{l z}} \Delta n_{l z}
\end{aligned}
$$

where, $h^{r}$ denotes the actual distance. Attentively, the above listed geometric parameters of the space robot amount to
32, but it does not mean that all these parameters can be identified, only for convenience. Equation(12) considers the influence of variation of the plane equation. Because $\mathbf{n}_{l}$ is a unit vector, the two of its three components are independent. Here we choose $n_{l y}$ and $n_{l z}$ as the parameters to be identified. Attentively, the parameter $f$ is unidentifiable, because $-f / n_{l z}$ represents the intercept that the plane intersects the coordinate $Z_{0}$ axis, and obviously it is associate with the parameter $d_{1}$. Of course, the above explanation assumes that the measured plane is not parallel to the $Z_{0}$ axis. Besides, the roughness of the plane will also weaken accuracy of the measurement, a good choice is that it is classified as measurement noise. The number of the identification equation must be greater than that of the identified geometric parameters. Obviously, only (12) is not enough. Simply, the more identification configurations are chosen to obtain the more identification equations. Through combining these equations the following formula can be given:

$$
\Delta \mathbf{h}=\mathbf{G} \Delta \mathbf{e}
$$

where $\Delta \mathbf{h}$ is the distance error vector, $\Delta \mathbf{h}=\left[\Delta h_{1} \Delta h_{2}\right.$ $\left.\cdots \Delta h_{m}\right], m$ denotes the $m$ th measurement configuration, $\Delta \mathbf{e}$ is the parameter error vector, $\Delta \mathbf{e}=\left[\Delta \theta_{1}, \Delta d_{1}\right.$, $\left.\Delta a_{1}, \Delta \alpha_{1}, \Delta \beta_{1}, \ldots, \Delta n_{l y}, \Delta n_{l z}\right], \mathrm{G}$ is the identification Jacobian matrix. According to (13), through iteration, we can identify the geometric parameters of the space robot and the measured plane.

\section{Simulation of Parameter Identification}

4.1. Optimal Experimental Design. The different measurement configurations have a certain impact on identification results. So, the selection of the measurement configurations is also important. At present, there are several proposed observability indexes to evaluate a set of measurement configurations. Since E-optimality is the best criterion to minimize the uncertainty of the end-effector pose of a robot and the variance of the parameters [20], it is used as the observability index of the optimal experimental design. Its objective function is to maximize the minimum singular value of the identification Jacobian matrix, and it can be written as

$$
\mathrm{O}_{3}=\max \sigma_{\min }(G)
$$

According to (14), when there are many sets of measurement configurations to be chosen, the set whose minimum singular value is maximal is the optimal experimental design.

4.2. Measurement Noise. There are usually some errors in the distance values measured by the laser-ranger, which will create disadvantageous effects on the geometric parameter identification of the space robot. In order to simulate the real case, measurement noise should be added to the error model so as to calibrate the space robot more exactly. Here, it is assumed that distance measurement noise follows a normal distribution with zero mean and standard deviation $0.2 \mathrm{~mm}$.

For the same configuration, the more distance measurements will be taken to reduce disturbance of the stochastic 
TABLE 1: Nominal D-H parameters of the space robot.

\begin{tabular}{lccccc}
\hline Link & $\theta_{n} / \mathrm{rad}$ & $\alpha_{n} / \mathrm{rad}$ & $a_{n} / \mathrm{m}$ & $d_{n} / \mathrm{m}$ & $\Delta \beta_{n} / \mathrm{rad}$ \\
\hline 1 & $\pi / 2$ & $-\pi / 2$ & 0 & 0.5 & - \\
2 & 0 & 0 & 1 & - & 0 \\
3 & $-\pi / 2$ & $\pi / 2$ & 0 & 0 & - \\
4 & 0 & $-\pi / 2$ & 0 & -0.8 & - \\
5 & $\pi$ & $\pi / 2$ & 0 & 0 & - \\
6 & 0 & 0 & -0.12 & 0.4 & 0 \\
\hline
\end{tabular}

Table 2: Pre-assumed geometrical Parameter Errors.

\begin{tabular}{lccccc}
\hline Link & $\Delta \theta_{n} / \mathrm{mrad}$ & $\Delta \alpha_{n} / \mathrm{mrad}$ & $\Delta a_{n} / \mathrm{mm}$ & $\Delta d_{n} / \mathrm{mm}$ & $\Delta \beta_{n} / \mathrm{mrad}$ \\
\hline 1 & -7.23 & -3.22 & 0.23 & 0.73 & - \\
2 & 0.52 & 0.13 & 1.94 & - & 1.45 \\
3 & 0.56 & -2.23 & 0.11 & 0.34 & - \\
4 & 0.36 & 1.92 & 0.18 & 1.35 & - \\
5 & -5.52 & -4.83 & 0.27 & 0.29 & - \\
6 & -0.34 & 0.62 & 0.47 & 0.85 & -3.36 \\
\hline
\end{tabular}

measurement noise, then the average of these measurements is provided as the measurand. On the other hand, the more redundant measurement configurations are used to identify the geometric parameters of the space robot, which has also an effect on filtering measurement noise.

4.3. Simulation Approach. According to the description above, the simulation approach of parameter identification of the space robot can be summarized as shown in Figure 3. Because the identification method is verified through simulation, a distance value calculated with the preassumed true parameters and the above-mentioned measurement noise will be used as a measurement value, and it is equal to the sum of the real value plus measurement noise. Besides, here, the estimated distance denotes the distance calculated with the nominal geometrical parameters.

4.4. Initial Condition. The nominal D-H parameters of the space robot are shown in Table 1 and its preassumed geometrical parameter errors are shown in Table 2.

In view of the space robot working on orbit lighted by the sun, the above length errors $\Delta a_{n}$ and $\Delta d_{n}$ are given a positive number in relation to their lengths, while the angle errors $\Delta \alpha_{n}, \Delta \beta_{n}$, and $\Delta \theta_{n}$ are given based on a normal distribution with zero mean and standard deviation $3.49 \mathrm{mrad}$. Attentively, the geometric parameters marked "-" in Table 2 are unidentifiable, so the identifiable parameters of the space robot amount to 25 .

Besides, the measured plane equation is chosen as

$$
y+4.6 z-0.69=0 .
$$

Attentively, as shown in Figure 1, the equation cannot be given such the form as $z+f=0$, or it will make three geometric parameters of the space robot unidentifiable, that is, $\theta_{1}, a_{1}, d_{3}$. Obviously, if the measured plane is perpendicular to the $Z_{0}$ axis, the three parameters will make no difference to the measured distance, which will weaken completeness of the identified geometric model. Because the measured plane expressed by (15) is parallel to the $X_{0}$ axis, for simplicity, here we only give the coefficient 4.6 an error, it is 0.005 .

4.5. Simulation Result. Subsequently, the above geometric parameter identification algorithm will be verified through simulation. Here, we have chosen 101 measurement configurations in all where the space robot is nonsingular. Then, the two cases will be simulated, namely, 50 configurations 10 repetitions (the first case) and 100 configurations 10 repetitions (the second case), $x$ repetitions denote the number of repeated measurements for a same measurement configuration. As stated in the Section 4.1, according to the optimal experimental design criterion, we will calculate $C_{101}^{100}$ minimum singular values of $\mathbf{G}$ for the first case, similarly for the second case, it is $C_{101}^{50}$ ones which are a huge number, and the task is difficult to come true. In fact, with the observability index $\mathrm{O}_{3}$, we calculate a part of the minimum singular values for the first case and all of them for the second case in simulation. According to the calculation results, the observability indexes of the above two cases are equal to 0.048 and 0.180 , respectively.

Besides, a set of independent validation configurations (20 configurations) distributing in the whole workspace of the space robot are selected to evaluate the identification effect. In nature, parameter identification is a fit for the measured data in the measurement configurations, so the extra validation configurations are necessary.

Figure 4 represents the distance errors in the measurement configurations, respectively, with the nominal parameters, the identified parameters for the first and second cases. It is easy to find that, after parameter identification, the maximum distance error in the measurement configurations decreases to less than $0.4 \mathrm{~mm}$ for the first case and to less than $0.2 \mathrm{~mm}$ for the second case, compared with more than $40 \mathrm{~mm}$ prior to parameter identification, so the parameter identification is a very good fit for the distance measurement values. At the same time, the maximum distance error with the identified parameters for the second case is less than that for the first case, which reflects the importance of more identification configurations. Of course, after identification, there still exist some fractional residual distance errors, which mainly come from measurement noise.

The position errors in the measurement configurations with the nominal, and the identified parameters for the first and second cases, are depicted in Figure 5, and the orientation errors are in Figure 6. Correspondingly, the position errors in the validation configuration are depicted in Figure 7, and the orientation errors are in Figure 8. In general, after parameter identification, pose accuracy of the space robot has a great improvement, for example, the position errors in the identification configuration are reduced from more than $15 \mathrm{~mm}$ to less than $1.5 \mathrm{~mm}$ for the second case and the orientation errors from $15 \mathrm{mrad}$ or so 




Figure 3: Simulation flowchart of parameter identification.

to $1.6 \mathrm{mrad}$ or so, especially in the validation configurations, it can be found that the position errors are reduced from $20 \mathrm{~mm}$ or so to less than $2 \mathrm{~mm}$ and the orientation errors from $20 \mathrm{mrad}$ or to less than $2.5 \mathrm{mrad}$. Besides, we noticed a law, namely, the pose errors in the identification configuration are fewer than those in the validation configuration, and the more the number of the identification configurations is, the higher the pose accuracy after identification is. In nature, parameter identification is a fit for measurement data in the identification configurations. However, it is an extrapolation in the validation configuration. So, the results in the identification configuration are better than those in the validation configurations. The observability index $\mathrm{O}_{3}$ in the more identification configurations is greater than that in the fewer identification configurations, so the identification results in the more identification configurations are better.

Tables 3 and 4, respectively, give the identified geometric parameter errors for the first and second case. In the two tables, the identified coefficient errors of the plane equation are not listed, and they are, respectively, 0.00243 and 0.00386 for the first and second case. Under the disturbance of measurement noise, these identified geometric parameter errors 


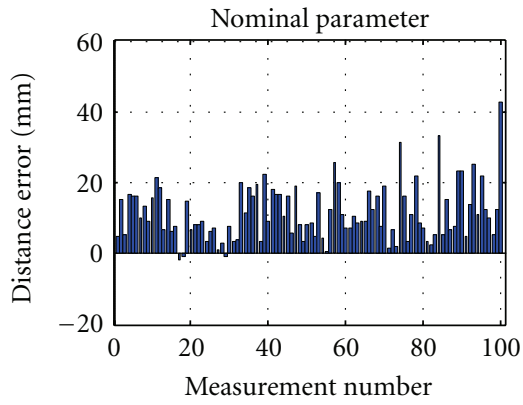

(a)

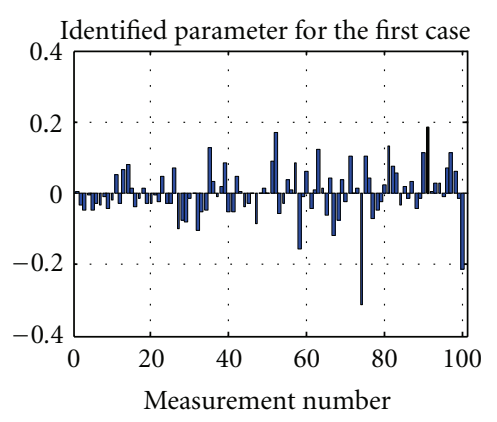

(b)

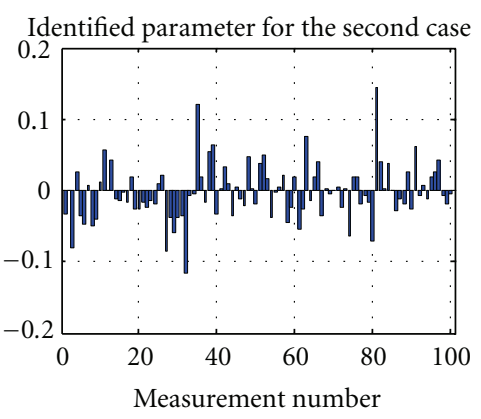

(c)

FIGURE 4: Distance errors prior to and after parameter identification.

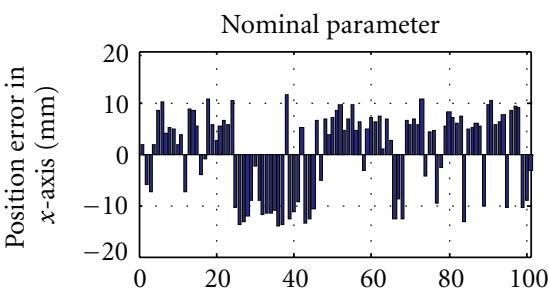

(a)

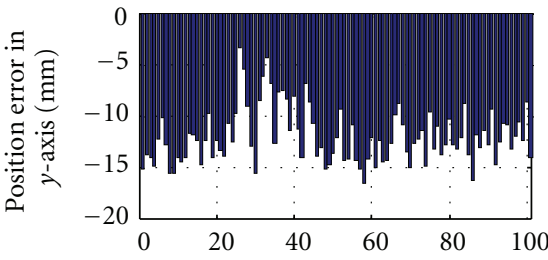

(d)

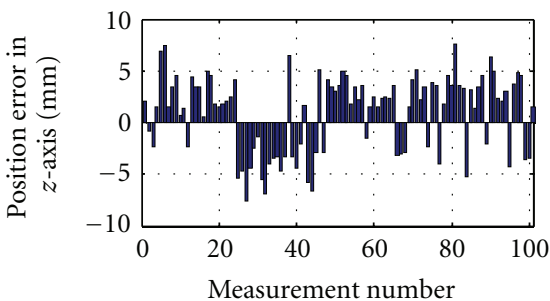

(g)

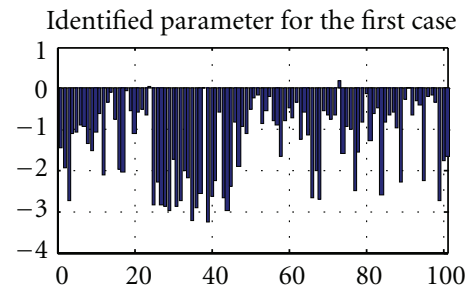

(b)

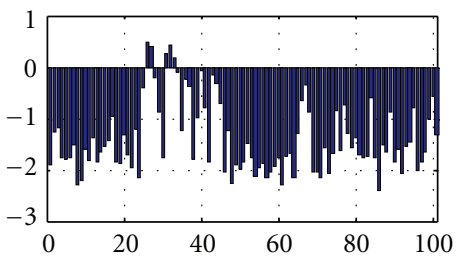

(e)



(h)

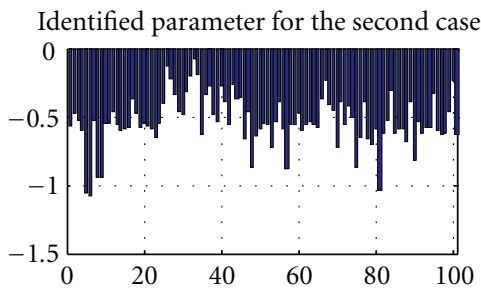

(c)

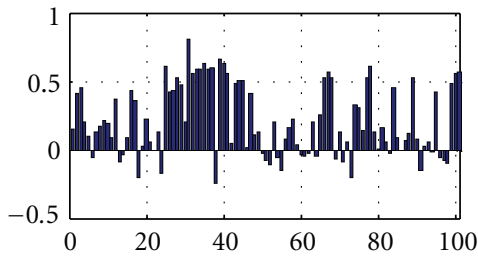

(f)

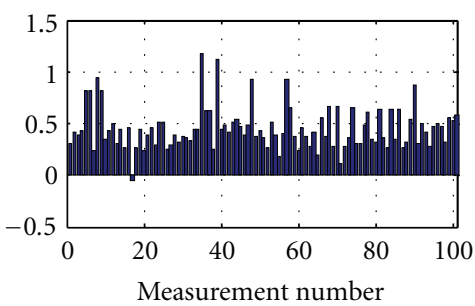

(i)

Figure 5: Position errors in the 101 identification configurations with the nominal, and the identified parameters for the first and second cases.

are inconsistent with the preassumed ones, but the identified parameter errors for the second case more approach them than those for the first case, which reflects that more measurement configurations can filter measurement noise better. If measurement noise is not added to the simulation, the identified parameters can match the preassumed parameters perfectly, which has been verified in the simulation.

Table 5 gives a statistical comparison of position and orientation errors calculated, respectively, with the nominal parameters, and the identified parameters for the first and second cases in the validation configurations. Here, RMS represents root mean square of pose errors, with respect to position or orientation error in the $x$ axis, it is written as

$$
\text { RMS_pose }=\sqrt{\frac{1}{m} \sum_{i=1}^{m}\left(\mathbf{p}_{r x}-\mathbf{p}_{x}\right)^{2}},
$$

where $\mathbf{p}_{r x}$ denotes the real position or orientation vector in the $x$ axis, and $\mathbf{p}_{x}$ is an estimated position or orientation vector with the nominal or identified parameters in the $x$ axis. The maximum position error denotes the maximum absolute position error value in the $x, y$, and $z$ axes, 


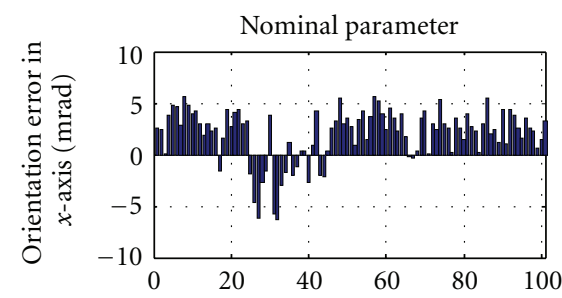

(a)

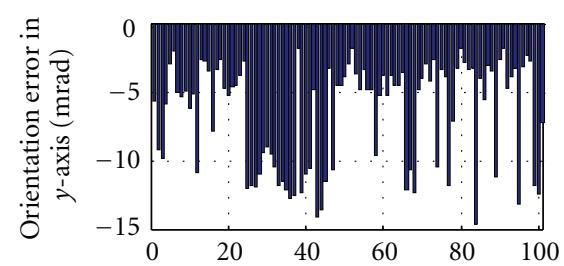

(d)

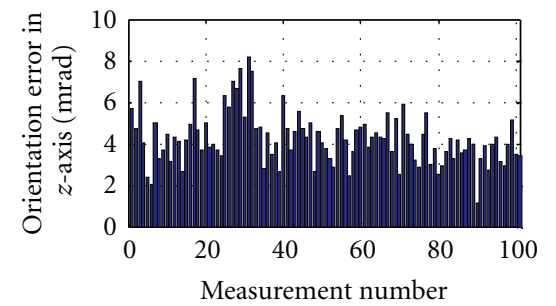

(g)

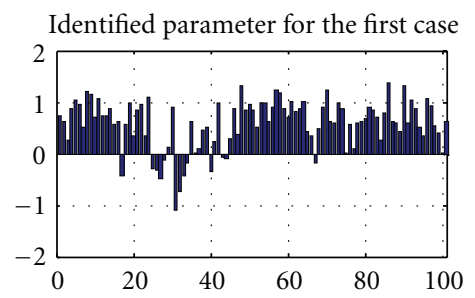

(b)

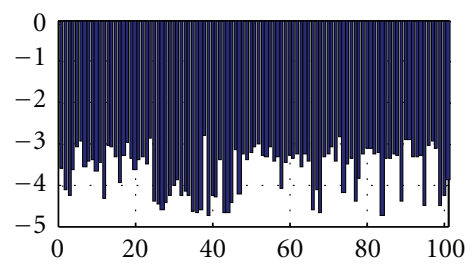

(e)

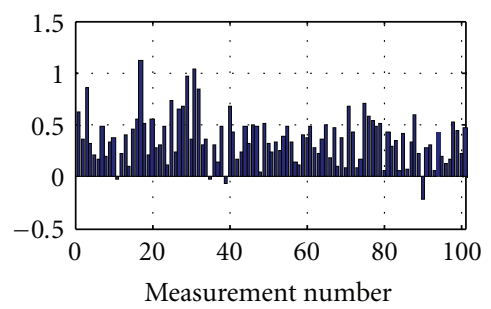

(h)

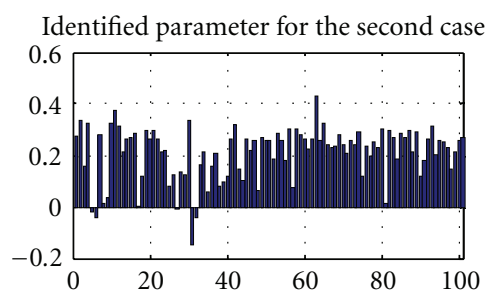

(c)

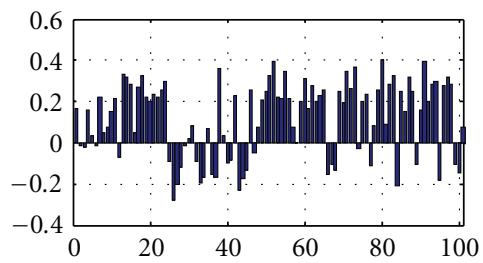

(f)

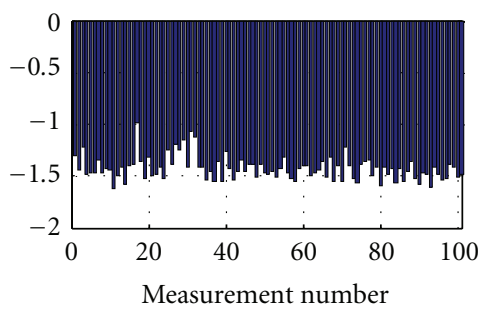

(i)

FIgURE 6: Orientation errors in the 101 identification configurations with the nominal, and the identified parameters for the first and second cases.

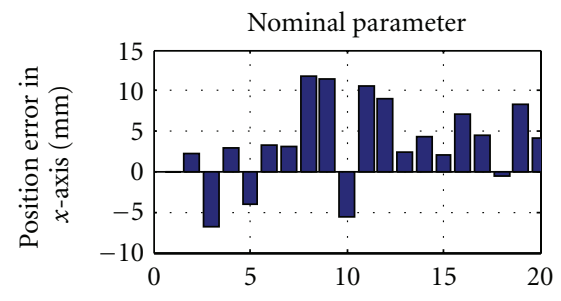

(a)

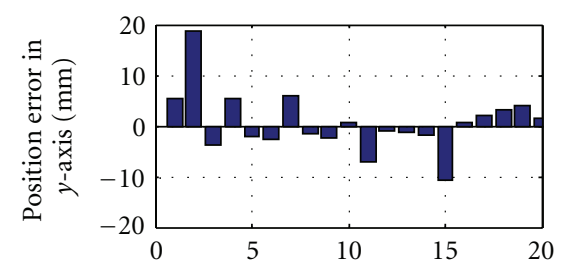

(d)

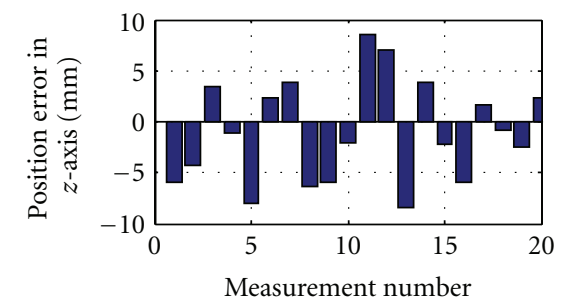

(g)

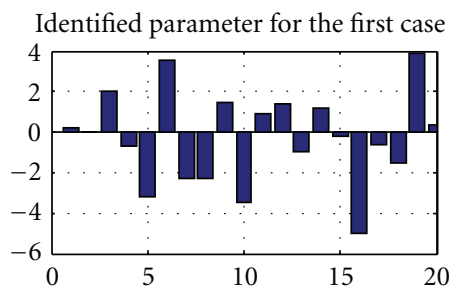

(b)

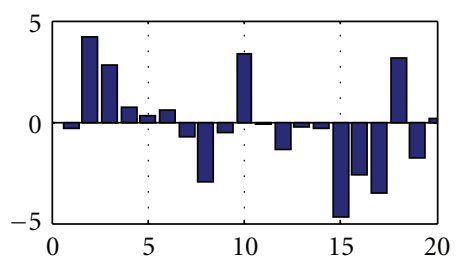

(e)



(h)

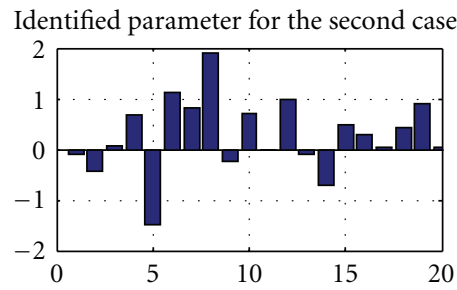

(c)

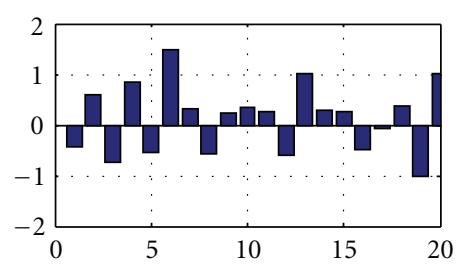

(f)

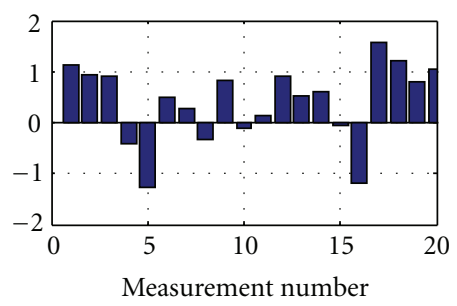

(i)

FIGURE 7: Position errors in the 20 validation configurations with the nominal, and the identified parameters for the first and second cases. 


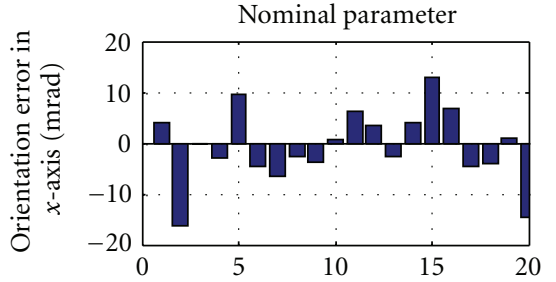

(a)

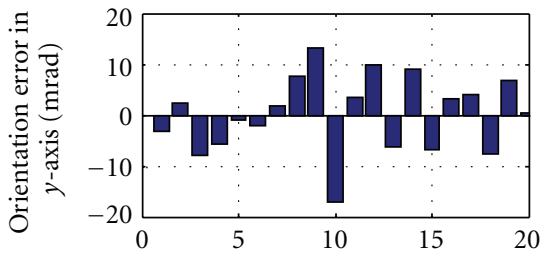

(d)

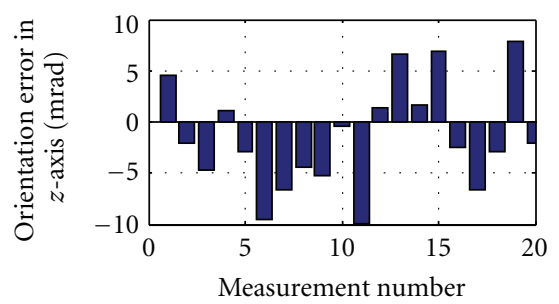

(g)

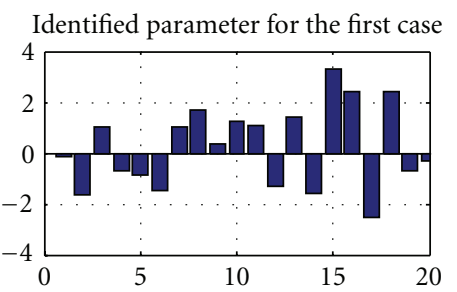

(b)

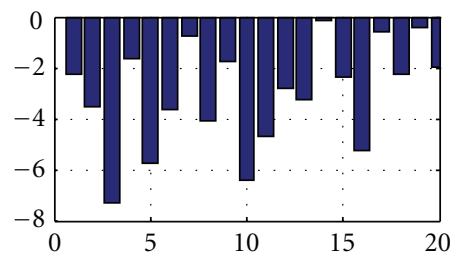

(e)



(h)

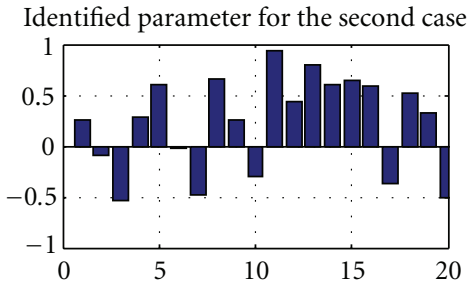

(c)

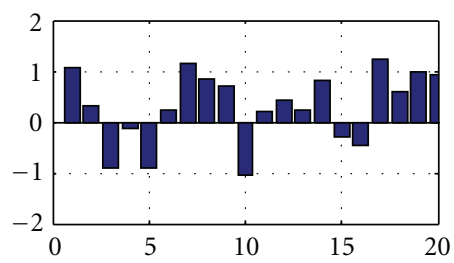

(f)

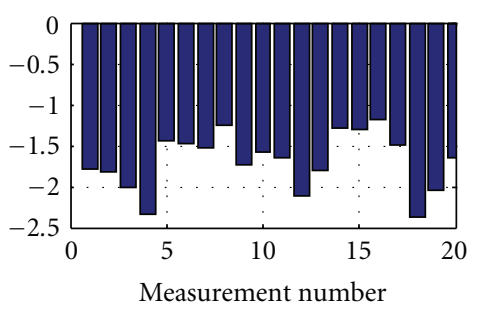

(i)

FIGURE 8: Orientation errors in the 20 validation configurations with the nominal, and the identified parameters for the first and second cases.

TABLE 3: Identified geometrical parameter errors for the first case.

\begin{tabular}{lccccc}
\hline Link & $\Delta \theta_{n} / \mathrm{mrad}$ & $\Delta \alpha_{n} / \mathrm{mrad}$ & $\Delta a_{n} / \mathrm{mm}$ & $\Delta d_{n} / \mathrm{mm}$ & $\Delta \beta_{n} / \mathrm{mrad}$ \\
Number & & & & & - \\
2 & -7.8525 & -2.4425 & -2.1491 & 0.5724 & - \\
3 & 1.2119 & 0.0553 & 1.6291 & - & 1.1436 \\
4 & 0.0949 & -1.5013 & 1.3863 & 0.2838 & - \\
5 & 0.2936 & 2.6439 & 0.3531 & 1.2179 & - \\
6 & -5.2130 & -3.8485 & -0.0797 & 0.2895 & - \\
\hline
\end{tabular}

and also for orientation errors. According to Table 5, it is found that improvement of pose accuracy after parameter identification is significant, and the maximum position error in the $y$ axis is reduced from $18.6857 \mathrm{~mm}$ to $1.4779 \mathrm{~mm}$ and the maximum orientation error from $17.0006 \mathrm{mrad}$ to $1.2271 \mathrm{mrad}$. Comparatively, the identification results for the second case are better than those for the first case as a whole, which shows that increment of the redundant measurement configurations can weaken disadvantageous influence of measurement noise and enhance identification effect. If more measurement configurations are added, better identification results can be expected.
TABLE 4: Identified geometrical parameter errors for the second case.

\begin{tabular}{lccccc}
\hline Link & $\Delta \theta_{n} / \mathrm{mrad}$ & $\Delta \alpha_{n} / \mathrm{mrad}$ & $\Delta a_{n} / \mathrm{mm}$ & $\Delta d_{n} / \mathrm{mm}$ & $\Delta \beta_{n} / \mathrm{mrad}$ \\
\hline 1 & -7.0607 & -2.8193 & -0.1191 & 0.1555 & - \\
2 & 0.8234 & 0.2574 & 1.6439 & - & 1.4527 \\
3 & 0.3428 & -2.0967 & 1.0172 & -0.5597 & - \\
4 & 0.5364 & 2.2958 & 0.0014 & 1.4096 & - \\
5 & -5.6786 & -4.2081 & 0.3607 & 0.6214 & - \\
6 & 1.4004 & 0.4974 & 0.4545 & 0.4274 & -3.3556 \\
\hline
\end{tabular}

\section{Conclusions}

(1) With the laser-ranger carried by the end effector the paper presents a geometric parameter identification method, and the 25 independent parameters of the space robot are identified through simulation. In the process of identification, independence of the parameters is discussed to avoid parameter dependence.

(2) Because space temperature environment also causes change of the measured plane, its coefficient needs also to be identified. In view of selection of the optimal measurement configurations, the observability index is used to evaluate the combinations of the measurement configurations, which 
TABLE 5: Comparison of position and orientation errors in the validation configuration.

\begin{tabular}{|c|c|c|c|c|c|}
\hline Error item & & $\begin{array}{l}\text { RMS position } \\
\text { error/mm }\end{array}$ & $\begin{array}{c}\text { RMS orientation } \\
\text { error/mrad }\end{array}$ & $\begin{array}{l}\text { Maximum position } \\
\text { error/mm }\end{array}$ & $\begin{array}{c}\text { Maximum orientation } \\
\text { error } / \mathrm{mrad}\end{array}$ \\
\hline \multirow{3}{*}{ Nominal parameter } & $x$ & 2.7612 & 3.1491 & 11.7347 & 16.1688 \\
\hline & $y$ & 2.5917 & 3.2196 & 18.6857 & 17.0006 \\
\hline & $z$ & 2.2119 & 2.3480 & 8.5899 & 9.9512 \\
\hline \multirow{3}{*}{ Identified parameter for the first case } & $x$ & 0.9921 & 0.7003 & 4.9654 & 3.2981 \\
\hline & $y$ & 1.0148 & 1.6199 & 4.6951 & 7.3035 \\
\hline & $z$ & 1.0524 & 0.5910 & 4.3151 & 2.7741 \\
\hline \multirow{3}{*}{$\begin{array}{l}\text { Identified parameter for the second } \\
\text { case }\end{array}$} & $x$ & 0.3427 & 0.2273 & 1.9067 & 0.9378 \\
\hline & $y$ & 0.2974 & 0.3374 & 1.4779 & 1.2271 \\
\hline & $z$ & 0.3785 & 0.7671 & 1.5664 & 2.3669 \\
\hline
\end{tabular}

reduces the possibility of inferior configurations to be introduced. At the same time, measurement noise of the laserranger is simulated to meet the actual state as much as possible.

(3) The simulation results show that in spite of distance measurement alone, the identification technique significantly improves pose accuracy of the space robot, which verifies the feasibility of the method.

\section{Acknowledgment}

This work is supported by National Nature Science Foundation of China (Nos. 60775049 and 60805033).

\section{References}

[1] W. K. Veitschegger and C. H. Wu, "Robot calibration and compensation," IEEE Journal of Robotics and Automation, vol. 4, no. 6, pp. 643-656, 1988.

[2] H. W. Stone, A. C. Sanderson, and C. P. Neuman, "Arm signature identification system," in Proceedings of the IEEE International Conference on Robotics and Automation, pp. 41-48, San Francisco, Calif, USA, 1986.

[3] H. W. Stone, A. C. Sanderson, C. P. Neuman et al., "A prototype arm signature identification system," in Proceedings of the IEEE International Conference on Robotics and Automation, pp. 175-182, Raleigh, NC, USA, 1987.

[4] L. Beyer and J. Wulfsberg, "Practical robot calibration with ROSY," Robotica, vol. 22, no. 5, pp. 505-512, 2004.

[5] Y. Sun and J. M. Hollerbach, "Active robot calibration algorithm," in Proceedings of the IEEE International Conference on Robotics and Automation (ICRA '08), pp. 1276-1281, May 2008.

[6] S. H. Kang, M. W. Pryor, and D. Tesar, "Kinematic model and metrology system for modular robot calibration," in Proceedings of the IEEE International Conference on Robotics and Automation, pp. 2894-2899, New Orleans, Fla, USA, May 2004.

[7] J. Chen and L. M. Chao, "Positioning error analysis for robot manipulators with all rotary joints," IEEE Transactions on Robotics and Automation, vol. 3, no. 6, pp. 539-545, 1987.

[8] R. P. Judd and A. B. Knasinski, "A technique to calibrate industrical robots with experimental verification," in Proceedings of the IEEE International Conference on Robotics and Automation, pp. 351-357, Raleigh, NC, USA, 1987.
[9] C. H. Gong, J. X. Yuan, and J. Ni, "Nongeometric error identification and compensation for robotic system by inverse calibration," International Journal of Machine Tools and Manufacture, vol. 40, no. 14, pp. 2119-2137, 2000.

[10] C. Lightcap, S. Hamner, T. Schmitz, and S. Banks, "Improved positioning accuracy of the PA10-6CE robot with geometric and flexibility calibration," IEEE Transactions on Robotics, vol. 24, no. 2, pp. 452-456, 2008.

[11] P. Drouet, S. Dubowsky, S. Zeghloul, and C. Mavroidis, "Compensation of geometric and elastic errors in large manipulators with an application to a high accuracy medical system," Robotica, vol. 20, no. 3, pp. 341-352, 2002.

[12] K. Radkhah, T. Hemker, and O. V. Stryk, "A novel self-calibration method for industrial robots incorporating geometric and nongeometric effects," in Proceedings of the IEEE International Conference on Mechatronics and Automation (ICMA '08), pp. 864-869, Takamatsu, Japan, August 2008.

[13] G. Hirzinger, K. Landzettel, B. Brunner et al., "DLR's robotics technologies for on-orbit servicing," Advanced Robotics, vol. 18, no. 2, pp. 142-144, 2004.

[14] G. Hirzinger, B. Brunner, J. Dietrich, and J. Heindl, "Sensorbased space robotics-ROTEX and its telerobotic features," IEEE Transactions on Robotics and Automation, vol. 9, no. 5, pp. 649-661, 1993.

[15] Y. Liu, Y. Shen, N. Xi et al., "Rapid robot/workcell calibration using line-based approach," in Proceedings of the 4th IEEE Conference on Automation Science and Engineering (CASE '08), pp. 510-515, Arlington, Va, USA, August 2008.

[16] C. S. Gatla, R. Lumia, J. Wood, and G. Starr, "Calibration of industrial robots by magnifying errors on a distant plane," in Proceedings of the IEEE/RSJ International Conference on Intelligent Robots and Systems (IROS '07), pp. 3834-3841, San Diego, Calif, USA, November 2007.

[17] I. W. Park, B. J. Lee, S. H. Cho, Y. D. Hong, and J. H. Kim, "Laser-based kinematic calibration of robot manipulator using differential kinematics," IEEE/ASME Transactions on Mechatronics, pp. 1-9, 2011.

[18] S. A. Hayati, "Robot arm geometric link parameter estimation," in Proceedings of the IEEE International Conference on Decision and Control, pp. 1477-1483, San Antonio, Tex, USA, 1983.

[19] L. Everett, M Driels, and B. Mooring, "Kinematic modeling for robot calibration," in Proceedings of the IEEE International Conference on Robotics and Automation, pp. 183-190, Raleigh, NC, USA, 1987. 
[20] Y. Sun and J. M. Hollerbach, "Observability index selection for robot calibration," in Proceedings of the IEEE International Conference on Robotics and Automation (ICRA '08), pp. 831836, Pasadena, Calif, USA, May 2008. 

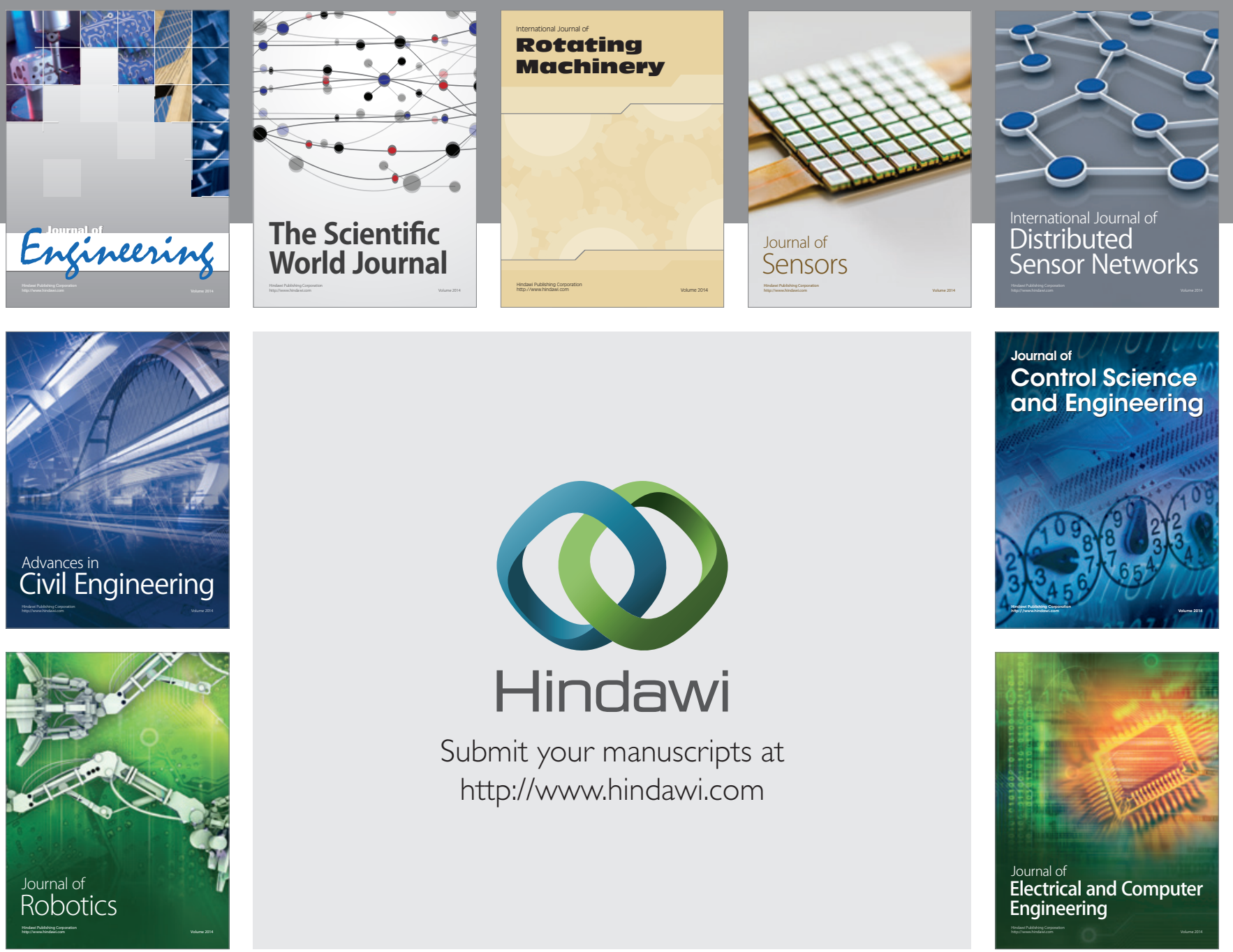

Submit your manuscripts at

http://www.hindawi.com
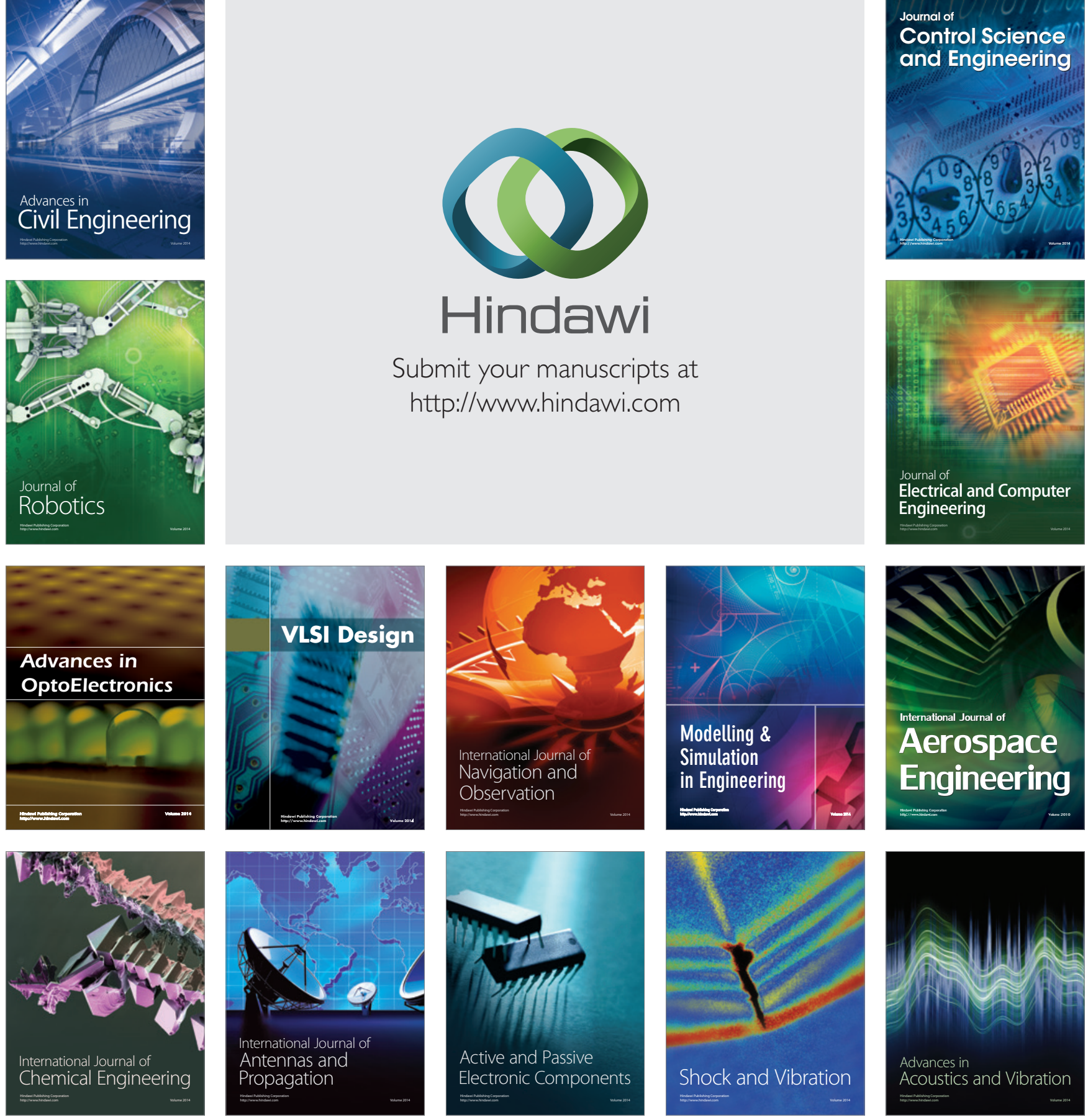